\title{
Transoral Incisionless Fundoplication for Refractory Gastroesophageal Reflux Disease: Where Do We Stand?
}

\author{
Deepanshu Jain ${ }^{1}$ and Shashideep Singhal ${ }^{2}$ \\ ${ }^{1}$ Department of Internal Medicine, Albert Einstein Medical Center, Philadelphia, PA, ${ }^{2}$ Division of Gastroenterology, Hepatology and Nutrition, \\ University of Texas Health Science Center at Houston, Houston, TX, USA
}

Gastroesophageal reflux disease (GERD) is a chronic, progressive, and costly medical condition affecting a substantial proportion of the world population, predominantly the Western population. The available treatment options for patients with refractory GERD symptoms are limited to either laparoscopic surgery with significant sequelae or potentially lifelong, high-dose proton pump inhibitor therapy. The restoration of the antireflux competence of the gastroesophageal junction at the anatomic and physiologic levels is critical for the effective long-term treatment of GERD. Transoral incisionless fundoplication (TIF) surgery is a safe, well-tolerated, and effective treatment that has yielded significant symptomatic improvement in patients with medically refractory GERD symptoms. In this review article, we have summarized case series and reports describing the role of TIF for patients with gastroesophageal reflux symptoms. The reported indications, techniques, complications, and success rates are also discussed. Clin Endosc 2016;49:147-156

Key Words: Endoscopic fundoplication; Gastroesophageal reflux; Proton pump inhibitors

\section{INTRODUCTION}

Gastroesophageal reflux disease (GERD) is a chronic, progressive, and costly medical condition affecting a substantial proportion of the world population, predominantly the Western population. The first clinical description of GERD appeared in 1935 as a case report on severe peptic esophagitis. ${ }^{1}$ GERD symptoms interrupt the activities of daily living and have been associated with significantly increased work absenteeism, reduced productivity at work, ${ }^{2-6}$ and increased healthcare resource utilization. ${ }^{6}$

During the previous years, many medical and surgical treatment options have been devised; however, they all are expen-

Received: March 10, 2015 Revised: May 19, 2015

Accepted: May 25, 2015

Correspondence: Shashideep Singhal

Division of Gastroenterology, Hepatology and Nutrition, University of Texas Health Science Center at Houston, 6431 Fannin, MSB 4.234, Houston, TX 77030 USA

Tel: +1-347-446-8203, Fax: +1-713-500-2107, E-mail: sdsinghal@gmail.com

(c) This is an Open Access article distributed under the terms of the Creative Commons Attribution Non-Commercial License (http://creativecommons.org/ licenses/by-nc/3.0) which permits unrestricted non-commercial use, distribution, and reproduction in any medium, provided the original work is properly cited. sive and pose significant adverse effects, which paved the way for the invention and advancement of effective endoscopic treatment options. Transoral incisionless fundoplication (TIF) is a unique form of natural orifice surgery, representing a next step in the field of minimally invasive surgery for the treatment of GERD.

TIF is an endoscopic luminal procedure that restores the antireflux competence of the gastroesophageal junction (GEJ), a critical step for effective long-term treatment of GERD. TIF is based on the principles of conventional antireflux surgery. It is done by using the EsophyX device (EndoGastric Solutions, Redmond, WA, USA), which is inserted transorally under endoscopic visual guidance to reconstruct the gastroesophageal valve (GEV) by wrapping the proximal part of the stomach (fundus) around the distal end of the esophagus, thus reestablishing the reflux barrier. The procedure involves placement of fasteners at four different positions to create $\mathrm{a} \geq 270^{\circ}$ valve that is 1 to $3 \mathrm{~cm}$ in length. Owing to this endoscopic incisionless approach, patients undergoing TIF experience less discomfort and faster recovery than those undergoing traditional antireflux surgery. Clinical studies have shown that TIF is an effective and safe treatment for mild to moderate GERD 
symptoms in carefully selected patients. The major and minor adverse events experienced with TIF compares favorably with those reported for laparoscopic fundoplication. ${ }^{7}$ TIF has helped patients in stopping acid-suppressive therapy, by maintaining or inducing the remission of GERD symptoms. ${ }^{8}$

In this review article, we have summarized case series and reports describing the use of TIF for GERD patients. The indications, techniques, clinical response, endoscopic response, limitations, and complications reported are also discussed.

\section{MATERIALS AND METHODS}

An extensive English-language literature search was done by using PubMed, Medline, and Google to identify peer-reviewed original and review articles by using the key terms "endoscopic fundoplication" and "GERD." Only articles on human patients were selected. The references of pertinent studies were manually searched to identify additional relevant studies. The indication, procedural details, technical and clinical success rates, complications, and limitations were considered part of the inclusion criteria. The search yielded mostly retrospective and prospective studies with a modest sample size, including case reports, case series, and randomized controlled trials.

None of the authors have any conflicts of interest or financial relationship with the company that produces or distributes the device described in the review article.

\section{RESULTS}

Ten original articles were considered appropriate for inclusion in this review. Among them, seven were prospective studies from the United States of America, ${ }^{8-10}$ Belgium, ${ }^{11}$ Netherlands, ${ }^{12,13}$ and Italy. ${ }^{14}$ Others included a retrospective study, ${ }^{15}$ a case report ${ }^{16}$ and a prospective sham controlled trial ${ }^{17}$ from the United States. All cases are summarized in Table 1.

\section{Indications}

Initially, TIF was approved by the Food and Drug Administration only for chronic GERD patients who are responsive to proton pump inhibitors (PPIs); however, over time, the inclusion criteria for TIF have expanded. Currently, the most common indication for TIF is either patients with refractory chronic GERD symptoms with only partial response to acid-suppressive medications, ${ }^{8-10,2-15,17}$ or those who do not want to continue lifelong medications despite being responsive to acid-suppressive medications. ${ }^{11,13,15}$ Kumta et al. ${ }^{16}$ described a unique case of TIF used for gastroesophageal reflux symptoms that developed after an endoscopic myotomy for underlying achalasia.

\section{Exclusion criteria}

The experience with TIF has grown in last few years but only to a specific segment of GERD patients. In nine of 10 studies included in our review, patients with a body mass index of $>35 \mathrm{~kg} / \mathrm{m}^{2}$, hiatal hernia $>2 \mathrm{~cm}$, grade D esophagitis according to the Los Angeles classification, esophageal motility disorder, and Barrett's esophagus were excluded from the study. ${ }^{8-15}$ A history of failed antireflux surgery was also one of the exclusion criteria in a few studies. ${ }^{11-13}$

\section{Technique}

The EsophyX device has been designed by Endogastric Solutions for the treatment of GERD. The use of the EsophyX2 device with a flexible endoscope has been the method of choice for most physicians performing TIF.

The procedure is performed under general anesthesia and requires the assistance of two physicians (surgeons and/or gastroenterologists). The first physician controls the EsophyX device; thus, monitoring the appropriate application of fasteners to secure the newly created GEV, and the second physician ensures continuous direct visualization with the endoscope. The device creates a GEV by retracting full-thickness plications and through the tailored placement of multiple fasteners circumferentially around the GEJ. ${ }^{18}$

There have been different versions of the TIF protocol with the major difference being in the degree of circumference of the reestablished valve, i.e., $220^{\circ}$ in the 1.0 protocol versus $240^{\circ}$ in the 2.0 protocol, and the location of the valve, i.e., at the level of the GEJ in the 1.0 protocol in contrast to the 2.0 protocol where the valve is created at a distance of 3 to $5 \mathrm{~cm}$ from the GEJ (Fig. 1). ${ }^{19}$ Cadière et al. ${ }^{11}$ described the use of the TIF 1.0 protocol in his study of 86 patients undergoing TIF with a mean wrap of $230^{\circ}\left(160^{\circ}\right.$ to $\left.300^{\circ}\right)$, whereas the TIF 2.0 protocol was used in most of the other studies ${ }^{8,10,13-15,17}$ with a minimum reported wrap of $240^{010,15}$ to as high as $300^{\circ} .{ }^{15} \mathrm{In}$ addition, the length of the reconstructed valve has been reported to vary from as low as $2 \mathrm{~cm}^{10,11,15}$ to as high as $6 \mathrm{~cm}^{11}$

\section{Clinical response}

The authors have used a wide variety of objective scales to quantify the GERD symptoms before and after TIF, such as the GERD health-related quality of life (GERD-HRQL), ${ }^{8,10-15}$ GERD symptom score (GERSS), ${ }^{10,15}$ reflux symptom index (RSI), ${ }^{8,10,15}$ reflux disease questionnaire (RDQ), ${ }^{8,17}$ and GERD quality of life (GERD-QUAL). ${ }^{14}$ Each of these scores, GERDHRQL, ${ }^{20}$ GERSS, ${ }^{21}$ RSI, ${ }^{22}$ RDQ, ${ }^{23}$ and GERD-QUAL, ${ }^{24}$ have been validated for assessing the GERD symptom severity and the response to the treatment. 


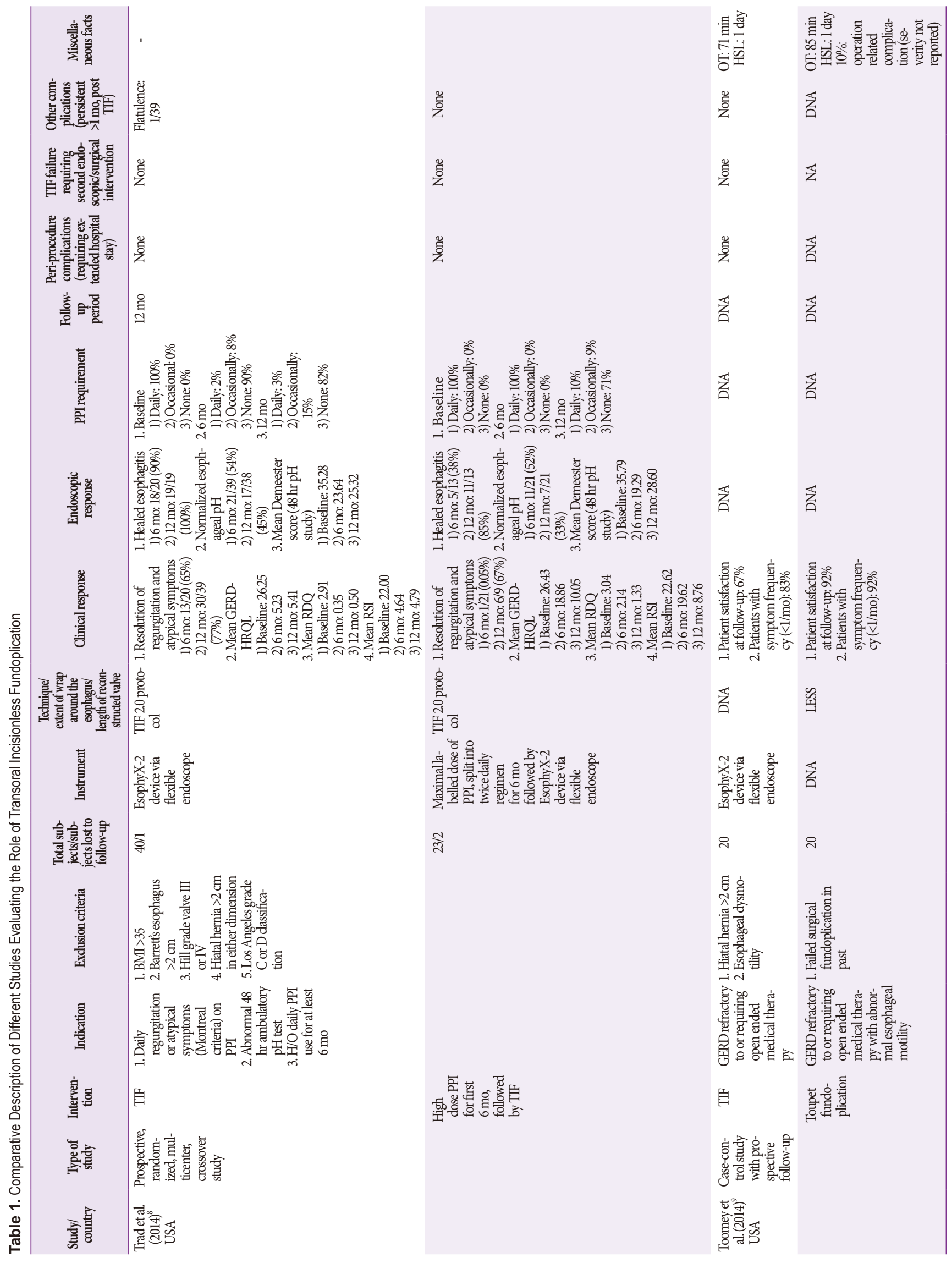




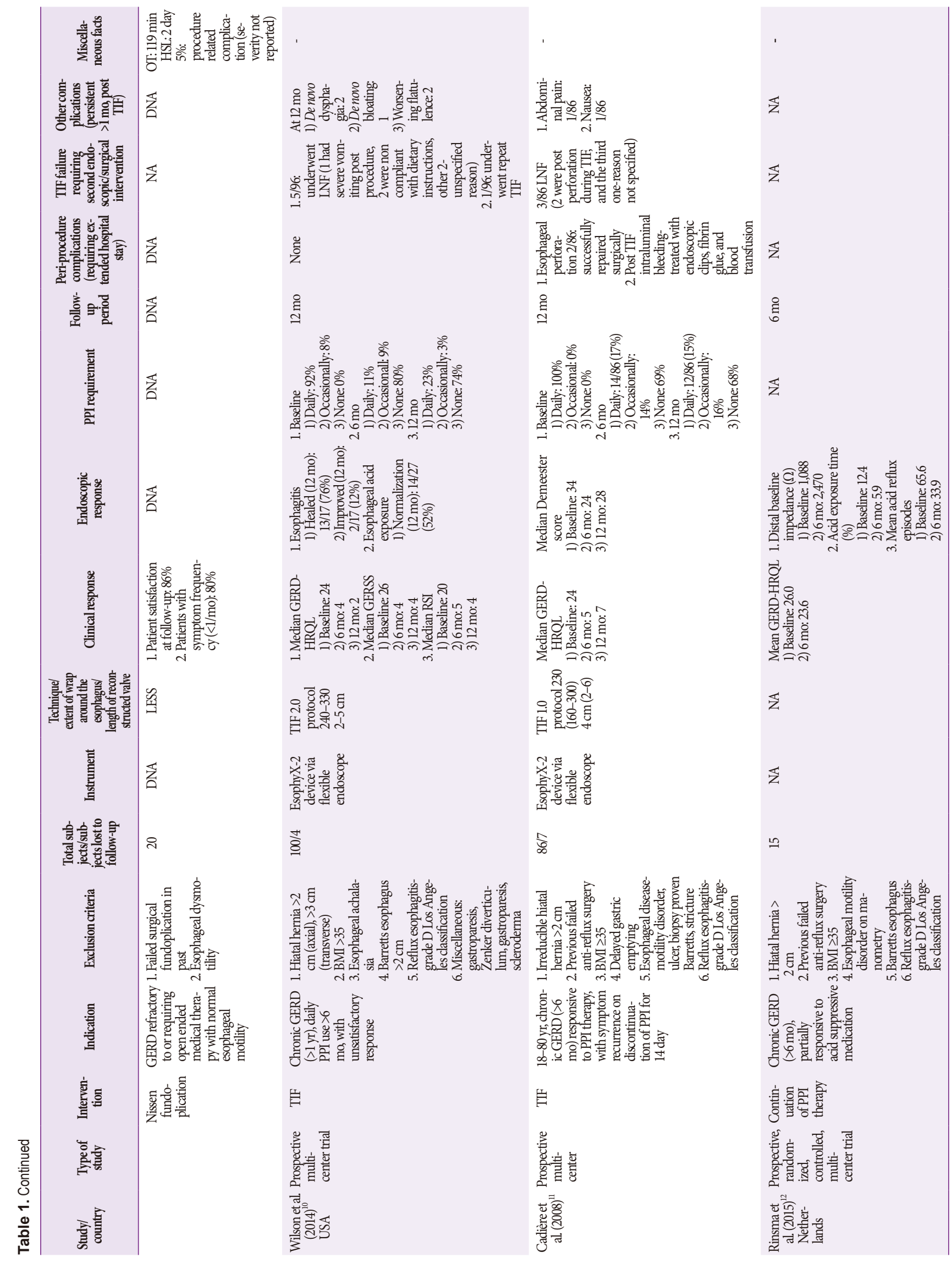




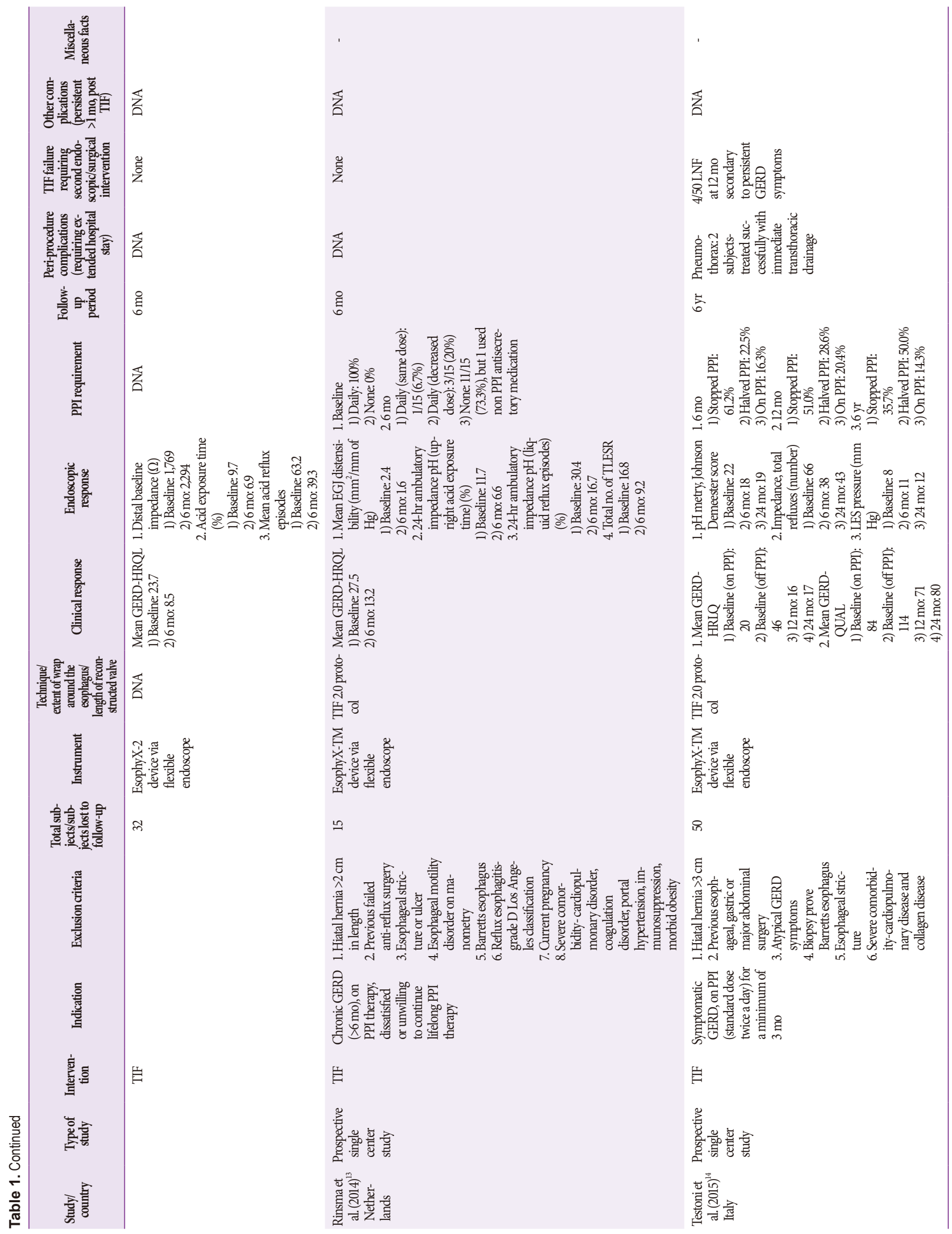




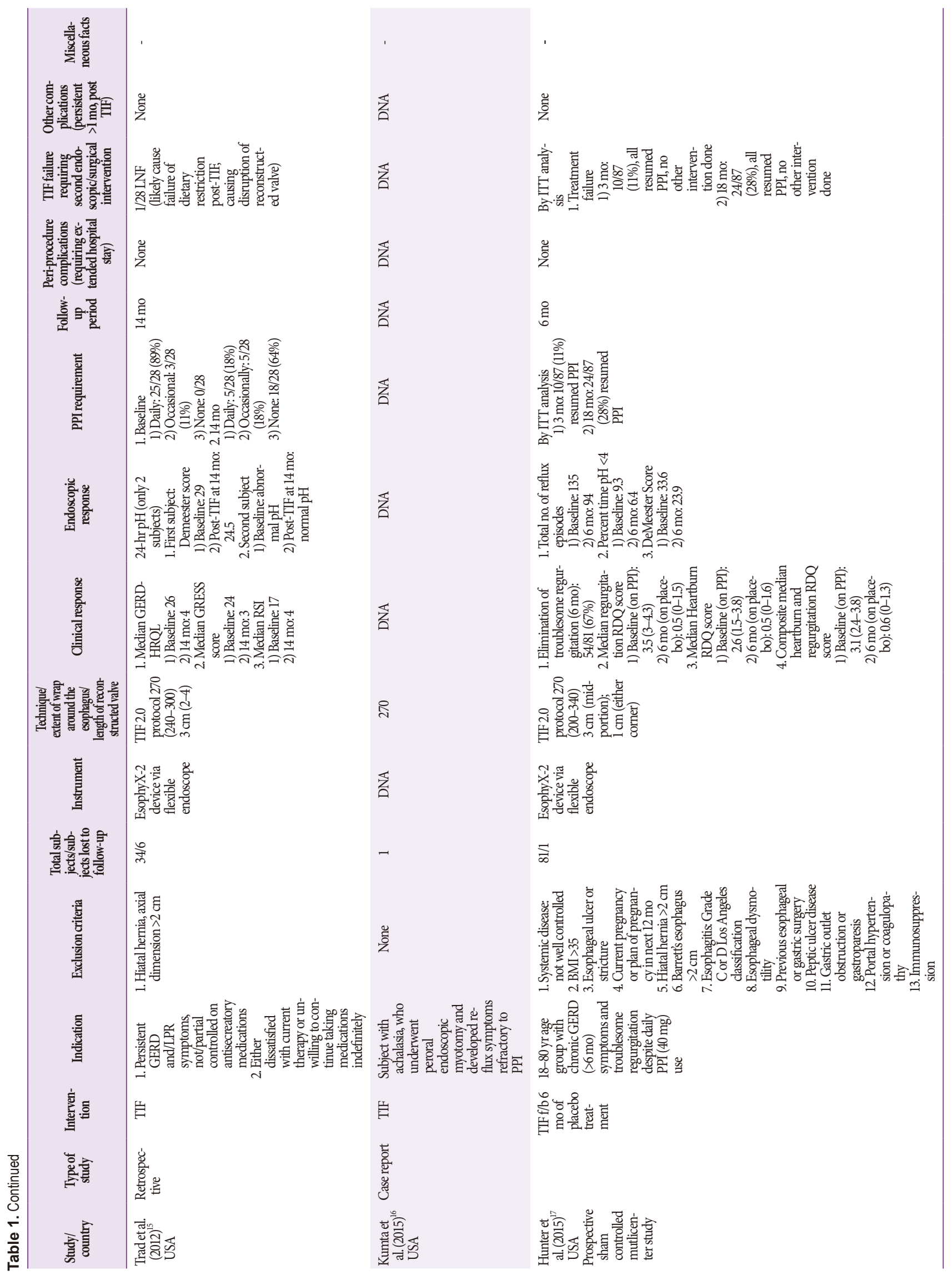




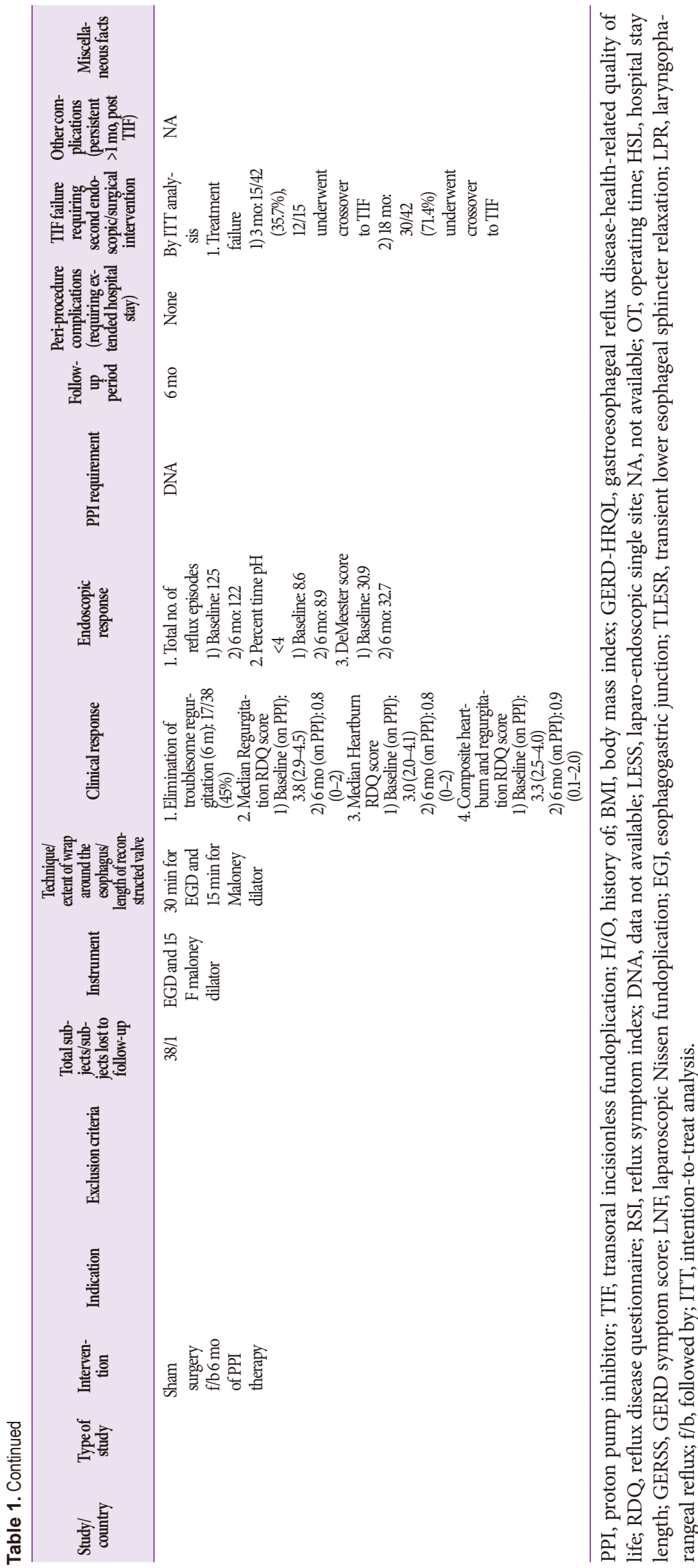

Trad et al. ${ }^{8}$ described a significant decrease in the mean GERD-HRQL (26.25 to 5.41), mean RDQ (2.91 to 0.50), and mean RSI scores (22.0 to 4.79) after TIF at 12 months follow-up. The study also showed that the TIF group (mean GERDHRQL [26.25 to 5.23], mean RDQ [2.91 to 0.35], and mean RSI scores [22.0 to 4.64]) showed better outcomes than the high-dose PPI group (mean GERD-HRQL [26.43 to 18.86], mean RDQ [3.04 to 2.14], and mean RSI scores [22.62 to 19.62]) at 6 months follow-up. The high-dose PPI group showed further decreases in mean GERD-HRQL (18.86 to 10.05), mean RDQ (2.14 to 1.33 ), and mean RSI scores (19.62 to 8.76) at 6 months after TIF. ${ }^{8}$ Toomey et al. ${ }^{9}$ described the patient satisfaction scores (67\%, 92\%, and 86\%) and the proportions of patients with a frequency of symptoms of $<1 /$ month $(83 \%, 92 \%$, and $80 \%)$ at postintervention follow-up across three subgroups (TIF, Toupet fundoplication, and Nissen fundoplication). Wilson et al. ${ }^{10}$ revealed a decrease in the median GERD-HRQL (24 to 2), median GERSS (26 to 4), and median RSI score (20 to 4) post-TIF at 12 months follow-up. Cadière et al. ${ }^{11}$ showed a decrease in the median GERD-HRQL scores from 12 to 7 at 12 months post-TIF. Rinsma et al. ${ }^{12}$ described a decrease in the mean GERDHRQL score from 23.7 to 8.5 post-TIF in contrast to almost similar results in the mean GERDHRQL scores (26.0 to 23.6) in the PPI study arm at 6 months follow-up. In another study, Rinsma et al. ${ }^{13}$ showed a similar trend in the mean GERDHRQL scores (27.5 to 13.2) post-TIF at 6 months follow-up. The study by Testoni et al. ${ }^{14}$ did not show a significant difference in GERD-HRQL (from 20 to 17) and GERD-QUAL (84 to 80) postTIF at 2 years follow-up. In another study by Trad et al., ${ }^{15}$ the results showed a decreasing trend in median GERD-HRQL (26.0 to 4), median RDQ (24 to 3), and median RSI scores (17 to 4) post-TIF at 14 months follow-up. Hunter et al., ${ }^{17}$ in a recent study, reported the elimination of troublesome regurgitation symptoms at 6 months follow-up in 54 of 81 patients (67\%) who underwent TIF and placebo treatment, in contrast to 17 of 38 patients (45\%) who underwent a sham procedure and PPI therapy. The trend in the median regurgitation RDQ score (3.5 to 0.5 ), median heartburn RDQ score (2.6 to 0.5 ), and composite median regurgitation and heartburn RDQ scores (3.1 to 0.6 ) 

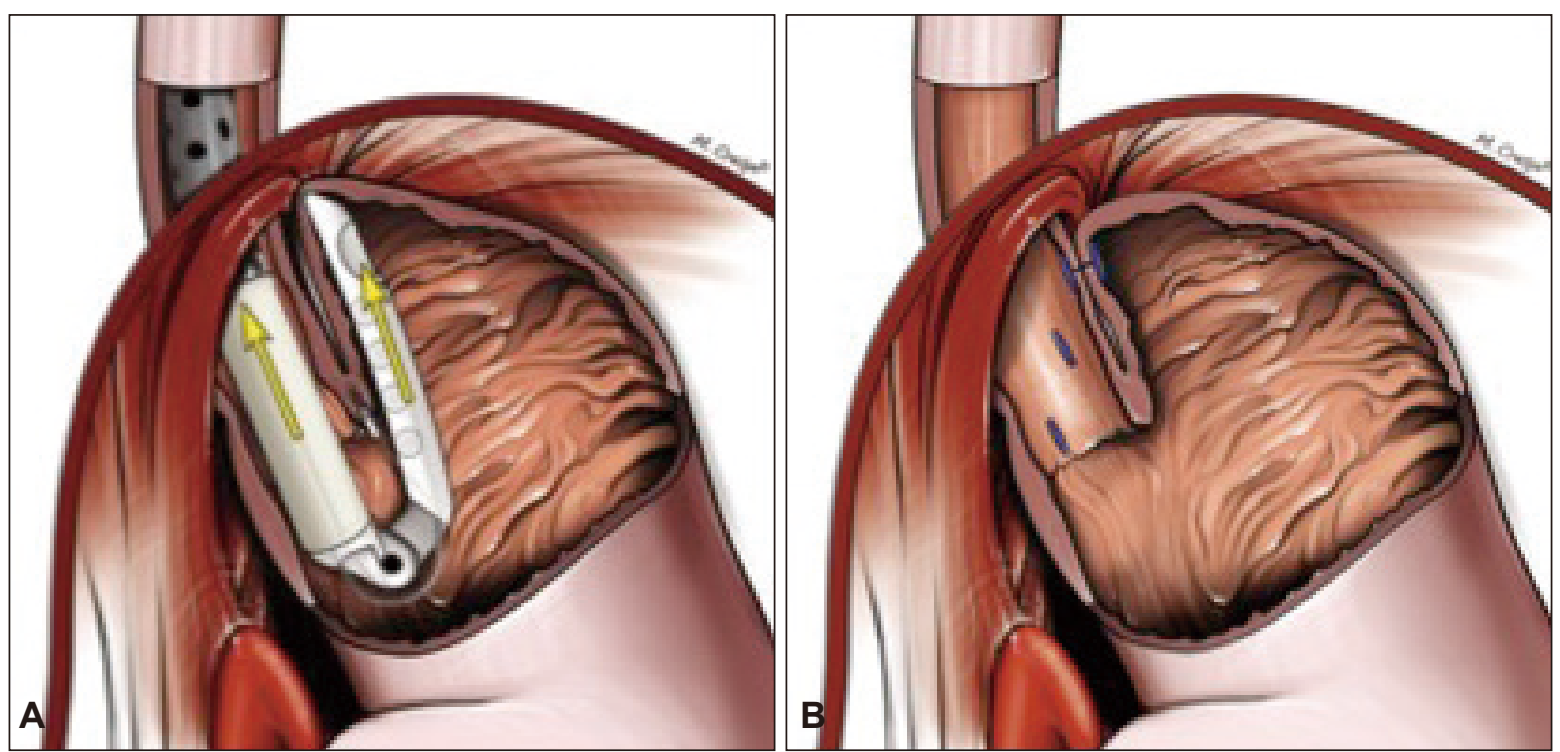

Fig. 1. (A) Creation of esophagogastric fundoplication using the EsophyX device (EndoGastric Solutions). (B) Post procedure appearance-esophagogastric fundoplication proximal to the Z-line. Adapted from Bell et al. ${ }^{19}$

among patients who underwent TIF and placebo medication was similar to that in patients who underwent a sham procedure and PPI treatment (median regurgitation RDQ score [3.8 to 0.8 ], median heartburn RDQ score [3.0 to 0.8 ], and composite median regurgitation and heartburn RDQ score [3.3 to 0.9]) at 6 months follow-up. ${ }^{17}$

Overall, the results of the above studies seem to be reflective of the improvement in the quality of life of GERD patients after TIF.

\section{Endoscopic response}

Trad et al..$^{8}$ described a significant decrease in the mean Demeester score (35.28 to 25.32) with normalization of esophageal $\mathrm{pH}$ in $45 \%$ (17 of 38 ) of patients, and healing of esophagitis in $100 \%$ of patients (19 of 19) post-TIF at 12 months follow-up. The study also showed that the TIF group (mean Demeester score [35.28 to 23.64] with normalization of esophageal $\mathrm{pH}$ in $54 \%$ of patients [21 of 39] and healed esophagitis in $90 \%$ of patients [18 of 20]) showed better outcomes than the high-dose PPI group (mean Demeester score [35.79 to 19.29] with normalization of esophageal $\mathrm{pH}$ in 52\% of patients [11 of 21] and healed esophagitis in only $38 \%$ of patients [5 of 13]) at 6 months follow-up. ${ }^{8}$ The high-dose PPI group showed further improvement in the proportion of patients with healed esophagitis (85\%, 11 of 13$)$ but a poor response reflected in the mean Demeester score (19.29 to 28.60) with normalization of esophageal pH in only $33 \%$ (7 of 21 ) of patients at 6 months after TIF. ${ }^{8}$ Wilson et al. ${ }^{10}$ reported normalization of esophageal acid exposure in 52\% (14 of 27) of patients with healed esophagitis in $76 \%$ of patients post-TIF at 12 months follow-up. Cadière et al. ${ }^{11}$ showed a decrease in the median Demeester score (34 to 28) post-TIF at 12 months follow-up. Rinsma et al. ${ }^{12}$ described an improvement in distal baseline impedance (1,769 to 2,294 $\Omega$ ) with a decrease in acid exposure time $(9.7 \%$ to $6.9 \%)$ and mean acid reflux episodes (63.2 to 39.3) in the post-TIF group, which was comparable to those in the PPI group (improvement in distal baseline impedance [1,088 to $2,470 \Omega$ ]; decrease in acid exposure time [12.4\% to $5.9 \%$ ] and mean acid reflux episodes [65.6 to 33.9]) at 6 months follow-up. In another study, Rinsma et al. ${ }^{13}$ showed an improvement in multiple endoscopically measured parameters, such as the mean GEJ distensibility (2.4 to 1.6 $\mathrm{mm}^{2} / \mathrm{mm} \mathrm{Hg}$ ), upright acid exposure time (11.7\% to $6.6 \%$ ), liquid reflux episodes (30.4\% to $16.7 \%$ ), and mean number of transient lower esophageal sphincter relaxation episodes (16.8 to 9.2) post-TIF at 6 months follow-up. Testoni et al. ${ }^{14}$ also reported a mild improvement in Demeester score (22 to 19) with a decrease in the total number of reflux episodes (66 to 43) and an increase in lower esophageal sphincter pressure ( 8 to $12 \mathrm{~mm} \mathrm{Hg}$ ) post-TIF at 2 years follow-up. Trad et al. ${ }^{15}$ reported only two patients at 14 months follow-up, with one showing normalization of esophageal $\mathrm{pH}$ and the other showing a decrease in Demeester score from 29 to 24.5. Hunter et al. ${ }^{17}$ reported a statistically significant $(p<0.001)$ improvement in the Demeester score (33.6 to 23.9), percent time with $\mathrm{pH}$ $<4$ (9.3\% to $6.4 \%$ ), and number of reflux episodes (135 to 94 ) in patients who underwent TIF and placebo treatment in contrast to those who underwent a sham procedure and PPI therapy (Demeester score [30.9 to 32.7], percent time with $\mathrm{pH}$ $<4$ [8.6\% to $8.9 \%$ ], and number of reflux episodes [125 to 122 ]) 
at 6 months follow-up.

Overall, the results of the studies seem to be reflective of effective esophageal mucosal healing in most of the GERD patients after TIF, thus indirectly decreasing the risk of the complications of chronic GERD.

\section{PPI requirement}

The proportion of patients requiring PPIs to control their GERD symptoms is one of the indirect measures of efficacy of the underlying intervention for GERD.

Trad et al. ${ }^{8}$ reported a significant decline in the proportion of daily PPI users (100\% to $3 \%$ ), with a concomitant increase in the proportion of occasional PPI users ( $0 \%$ to $15 \%)$ and those who do not use PPIs ( $0 \%$ to $82 \%$ ) post-TIF at 12 months follow-up. The high-dose PPI group also showed a similar trend in the proportion of daily PPI users (100\% to $10 \%)$ with a concomitant increase in the proportion of occasional PPI users ( $0 \%$ to $9 \%$ ) and those who remain not using PPIs ( $0 \%$ to $71 \%)$ at 6 months after TIF. ${ }^{8}$ Wilson et al. ${ }^{10}$ reported a decline in the proportion of daily PPI users (92\% to $23 \%$ ) and occasional PPI users (8\% to $3 \%$ ) with concomitant increase in patients who remain not using PPIs ( $0 \%$ to $74 \%$ ) after TIF at 12 months follow-up. Cadière et al. ${ }^{11}$ also showed a decline in the proportion of daily PPI users (100\% to $15 \%$ ) with a concomitant increase in the proportion of occasional PPI users ( $0 \%$ to $16 \%)$ and those who remain not using PPIs ( $0 \%$ to $68 \%$ ) after TIF at 12 months follow-up. Rinsma et al. ${ }^{13}$ showed a similar trend, with 11 of 15 patients (73.3\%) stopping their PPI use altogether and the remaining 4 of 15 users (26.7\%) reporting daily use, but with 3 of the 4 daily users not able to decrease the daily dose of PPI, at 6 months after TIF. The study by Testoni et al. ${ }^{14}$ was unique in comparison to the rest of the studies because of its long follow-up of 6 years. The results showed that after TIF, the proportion of daily PPI users decreased at 6 months (100\% to $16.3 \%)$ and at 6 years $(16.3 \%$ to $14.3 \%)$ follow-up. There was a concomitant increase in patients who were using half the PPI dose at 6 months ( $0 \%$ to $22.5 \%)$ and at 6 years $(22.5 \%$ to $50.0 \%)$ follow-up. ${ }^{14}$ In addition, the proportion of patients who have stopped PPI initially increased ( $0 \%$ to $61.2 \%)$ and then decreased (61.2\% to $35.7 \%$ ) at 6 years follow-up. ${ }^{14}$ In another study, Trad et al. ${ }^{15}$ reported a decreasing trend in the proportion of daily PPI users (89\% to $18 \%$ ) with a concomitant increase in the proportion of occasional PPI users (11\% to $18 \%$ ) and those who remain not using PPIs ( $0 \%$ to $64 \%$ ) post-TIF at 14 months follow-up. Hunter et al. ${ }^{17}$ reported that of 87 patients who underwent TIF with placebo treatment, 10 patients (11\%) at 3 months follow-up and 24 patients (28\%) at 18 months follow-up resumed PPI therapy because of the failure to resolve the GERD symptoms.

Overall, the results of the above studies are reflective of a significant decrease in the proportion of GERD patients requiring PPI after TIF, which indirectly has a major impact in decreasing the incidence of PPI-related complications among these patients.

\section{Follow-up}

Most authors reported a follow-up period ranging from a minimum of 6 months ${ }^{12,13,17}$ to a maximum of 6 years. ${ }^{14} \mathrm{~A}$ total of 575 patients were studied in the 10 studies included in our review, among whom 22 patients were lost to follow-up. Despite a good follow-up, many patients with a successful control of symptoms after fundoplication might still be compliant to clinical questionnaire surveys on return visits but are nearly universally noncompliant to follow-up pH monitoring, thus leaving fewer patients for a comparison of the endoscopic outcomes.

\section{TIF failure}

Of the 575 total patients, 492 underwent TIF, 14 (2.84\%) of whom required a repeat intervention. One patient underwent a repeat TIF procedure ${ }^{10}$ and the other 13 required laparoscopic fundoplication. ${ }^{10,11,14,15}$ Of these 13 cases, four were secondary to persistent GERD symptoms, ${ }^{14}$ two were secondary to esophageal perforation post-TIF, ${ }^{11}$ three were secondary to noncompliance to post-TIF dietary recommendations, ${ }^{10,15}$ one was secondary to severe post-TIF vomiting, ${ }^{10}$ and the other three had unknown etiology. ${ }^{10,11}$ Hunter et al..$^{17}$ reported TIF failure in 10 of $87(11 \%)$ patients at 3 months and 24 of $87(28 \%)$ patients at 18 months follow-up, necessitating the resumption of PPI use; however, none of the patients underwent a repeat TIF or laparoscopic surgery. In the same study, Hunter et al. ${ }^{17}$ reported that among the group who underwent sham surgery with placebo medication, 15 of 42 patients (35.7\%) at 3 months and 30 of 42 patients (71.4\%) at 18 months follow-up underwent TIF for persistent GERD symptoms.

\section{Periprocedural complications (requiring extended hospital stay)}

Of the 575 total patients, 492 underwent TIF, five (1.01\%) of whom required an extended hospital stay secondary to the procedure-related complication. Of these five patients, two had esophageal perforation that required surgical treatment; ${ }^{11}$ two had pneumothorax that was treated with transthoracic drainage; ${ }^{14}$ and the other one had gastrointestinal bleeding that was treated with endoscopic clips, fibrin glue, and supportive blood transfusion. ${ }^{11}$

\section{Long-term procedure-related adverse effects (per- sistent/de novo $>1$ month post-TIF)}

Of the 575 total patients, 492 underwent TIF, of whom 
only eight (1.62\%) were reported to have had symptoms that persisted beyond 1 month after TIF, or completely new symptoms that appeared 1 month after TIF that were attributed to the procedure. Three patients had worsening flatulence at 12 months follow-up, ${ }^{8,10}$ two had de novo dysphagia at 12 months follow-up, ${ }^{10}$ one had de novo bloating at 12 months follow-up, ${ }^{10}$ one had persistent abdominal pain at 1 month follow-up, ${ }^{11}$ and the other one had persistent nausea at 1 month follow-up. ${ }^{11}$

\section{CONCLUSIONS}

TIF for chronic GERD can be a safe, minimally invasive, and equally efficacious alternative approach to surgery in selected patients who have refractory symptoms or are reluctant to take lifelong acid-suppressive medications or have contraindications to surgery. With the evolving technique and increasing experience, TIF seems to be a reasonable firstline approach for the management of a specific subgroup of patients with chronic GERD. The preliminary reports appear promising; however, larger multicentric prospective randomized sham-controlled trials with a longer follow-up and head-to-head comparison between PPI and other modalities for the treatment of GERD are needed in the future to ascertain its benefits before it can be adopted as a standard alternative therapy for patients with chronic GERD.

\section{Conflicts of Interest}

The authors have no financial conflicts of interest.

\section{Acknowledgments}

D.J. performed literature search, data collection, compilation of results, and writing of the manuscript. S.S. performed literature search, and edited the manuscript.

\section{REFERENCES}

1. Dent J. Landmarks in the understanding and treatment of reflux disease. J Gastroenterol Hepatol 2009;24 Suppl 3:S5-S14.

2. Dent J, El-Serag HB, Wallander MA, Johansson S. Epidemiology of gastro-oesophageal reflux disease: a systematic review. Gut 2005;54:710717.

3. Heidelbaugh JJ, Gill AS, Van Harrison R, Nostrant TT. Atypical presentations of gastroesophageal reflux disease. Am Fam Physician 2008; $78: 483-488$

4. Fass R. Effect of gastroesophageal reflux disease on sleep. J Gastroenterol Hepatol 2010;25 Suppl 1:S41-S44

5. Friedenberg FK, Hanlon A, Vanar V, et al. Trends in gastroesophageal reflux disease as measured by the National Ambulatory Medical Care Survey. Dig Dis Sci 2010;55:1911-1917.
6. Toghanian S, Wahlqvist P, Johnson DA, Bolge SC, Liljas B. The burden of disrupting gastro-oesophageal reflux disease: a database study in US and European cohorts. Clin Drug Investig 2010;30:167-178.

7. Reavis KM, Perry KA. Transoral incisionless fundoplication for the treatment of gastroesophageal reflux disease. Expert Rev Med Devices 2014;11:341-350.

8. Trad KS, Simoni G, Barnes WE, et al. Efficacy of transoral fundoplication for treatment of chronic gastroesophageal reflux disease incompletely controlled with high-dose proton-pump inhibitors therapy: a randomized, multicenter, open label, crossover study. BMC Gastroenterol 2014;14:174.

9. Toomey P, Teta A, Patel K, Ross S, Sukharamwala P, Rosemurgy AS. Transoral incisionless fundoplication: is it as safe and efficacious as a Nissen or Toupet fundoplication? Am Surg 2014;80:860-867.

10. Wilson EB, Barnes WE, Mavrelis PG, et al. The effects of transoral incisionless fundoplication on chronic GERD patients: 12-month prospective multicenter experience. Surg Laparosc Endosc Percutan Tech 2014:24:36-46.

11. Cadière GB, Buset M, Muls V, et al. Antireflux transoral incisionless fundoplication using EsophyX: 12-month results of a prospective multicenter study. World J Surg 2008;32:1676-1688.

12. Rinsma NF, Farré R, Bouvy ND, Masclee AA, Conchillo JM. The effect of endoscopic fundoplication and proton pump inhibitors on baseline impedance and heartburn severity in GERD patients. Neurogastroenterol Motil 2015;27:220-228.

13. Rinsma NF, Smeets FG, Bruls DW, et al. Effect of transoral incisionless fundoplication on reflux mechanisms. Surg Endosc 2014;28:941-949.

14. Testoni PA, Testoni S, Mazzoleni G, Vailati C, Passaretti S. Long-term efficacy of transoral incisionless fundoplication with Esophyx (Tif 2.0) and factors affecting outcomes in GERD patients followed for up to 6 years: a prospective single-center study. Surg Endosc 2015;29:2770-2780.

15. Trad KS, Turgeon DG, Deljkich E. Long-term outcomes after transoral incisionless fundoplication in patients with GERD and LPR symptoms. Surg Endosc 2012;26:650-660.

16. Kumta NA, Kedia P, Sethi A, Kahaleh M. Transoral incisionless fundoplication for treatment of refractory GERD after peroral endoscopic myotomy. Gastrointest Endosc 2015;81:224-225.

17. Hunter JG, Kahrilas PJ, Bell RC, et al. Efficacy of transoral fundoplication vs omeprazole for treatment of regurgitation in a randomized controlled trial. Gastroenterology 2015;148:324-333.e5.

18. Cadière GB, Rajan A, Germay O, Himpens J. Endoluminal fundoplication by a transoral device for the treatment of GERD: a feasibility study. Surg Endosc 2008;22:333-342.

19. Bell RC, Cadière GB. Transoral rotational esophagogastric fundoplication: technical, anatomical, and safety considerations. Surg Endosc 2011;25:2387-2399.

20. Velanovich V. The development of the GERD-HRQL symptom severity instrument. Dis Esophagus 2007;20:130-134.

21. Allen CJ, Parameswaran K, Belda J, Anvari M. Reproducibility, validity, and responsiveness of a disease-specific symptom questionnaire for gastroesophageal reflux disease. Dis Esophagus 2000;13:265-270.

22. Belafsky PC, Postma GN, Koufman JA. Validity and reliability of the reflux symptom index (RSI). J Voice 2002;16:274-277.

23. Shaw M, Dent J, Beebe T, et al. The Reflux Disease Questionnaire: a measure for assessment of treatment response in clinical trials. Health Qual Life Outcomes 2008;6:31.

24. Chan Y, Ching JY, Cheung CM, et al. Development and validation of a disease-specific quality of life questionnaire for gastro-oesophageal reflux disease: the GERD-QOL questionnaire. Aliment Pharmacol Ther 2010;31:452-460. 